\title{
Automatic Maintenance Routes Based on the Quality Assurance Information
}

\author{
Vesa Hasu and Heikki Koivo \\ Aalto University, Department of Automation and Systems Technology \\ Finland
}

\section{Introduction}

Maintenance and repairing of widely distributed and large scale networks of sensors and actuators can easily become overwhelming - physically and financially. This applies especially, if the units are located so that performing the maintenance operations is neither easily accessible nor cheap. In order to make effective and efficient maintenance decisions, we need methodologies for describing the condition of the units. For that purpose, we propose using general quality concept descriptions. This methodology links linguistic terms and numerical values into easily understandable concepts on the network performance.

The market interest in large-scale networks has risen with the ability to utilize wireless communication nodes. The arranging of the maintenance of such large-scale networks becomes tedious due to sheer size of the problem. In this work, we assume a network, which is distributed spatially in wide area. That is, the movement of maintenance personnel takes much time and physically this means that repairing every occurring fault is too time timeconsuming. Economically this means that the distances are large enough to cause significant costs. Statistically "widely distributed" means that measurement stations next to each other are not exactly valid reference measurements for reliability estimation. In overall, any automated help is welcome to making automated maintenance decisions.

In our approach, we take the essential thing is to express the operation of a device in a simple numerical form of one index and analyze device maintenance needs based on the numerical value. In general we consider this index as reliability. In here, the reliability refers specifically to the unit performance reliability. That is, this reliability refers to a larger concept than an occurrence fault failure. This is because, for example, in the measurement networks an exact detection of faults is impossible or fuzzyfied.

In Isermann (2006) reliability is defined as the "ability of a system to perform a required function under stated conditions, within a given scope, during a given period of time" and availability is defined as "probability that a system or equipment will operate satisfactorily and effectively at any period of time." These definitions apply at some level in this work too, but since the errors are not exactly traceable, we aim to use performance indices to describe these concepts instead of e.g. failure probabilities or failure densities. Hence, in our work, the proposed concepts of reliability and availability are described by indices varying between 0 and 1 . We emphasize that these indices are not probabilities; they are measures of performance in the index framework. 
The reliability in a spatially distributed system is considered e.g. by Sun et al. (2005), which approaches the reliability through redundant measurements and voting procedures. In here the redundancy of measurements is not assumed, only through the spatial dependency of the measurement field.

De Souza et. al. (2005) explore monitoring of power networks with a fitness function methodology, which include the critical system measurement weighting. In the context of logistics systems, spatially distributed systems are considered by Wang et al. (2006), who describe accurate mathematical definitions of reliability.

In here, more emphasis is laid on describing the overall ideas in measurement networks and going through mathematical details by a case example on weather station networks. This chapter is based on the performance indices to the measurements networks presented by Hasu and Koivo $(2007,2009)$ but we consider new and improved methods for route solving. Additionally, we emphasize that the automatic maintenance route approach can be utilized in actuator networks. In this case, the performance indices used for industrial plant level by Hölttä and Koivo $(2009,2011)$ are interesting.

This chapter studies application of the quality assurance knowledge in the automatic maintenance route planning. The original framework is aimed at sensor network maintenance but the basic ideas of route planning are transferable also into actuator networks. The suggested approach has three essential stages: the basic quality assurance, the evaluation of performance indices and the maintenance route planning.

At first, quality assurance techniques determine whether sensors and/or processes work correctly or suffer from suspicious behavior. Second, specially designed performance indices are applied to describe the observation history. The key is to describe the reliability in one index as well as possible. The strengths of the performance index approach include that it enables forgiving bad functioning periods. If the problems are gone, maintenance actions for that component are futile and they result in unnecessary costs. The second main strength of performance indices is the ability to give a quick overlook on the performance without a need to see the whole history with its QA information. The third stage of our approach is the automatic planning of maintenance routes based on performance indices. Since this route-planning problem is close to the travelling salesman problem, routes are solved using either heuristic or evolutionary computing methods using somewhat similar ideas. This chapter demonstrates the approach using surface weather stations.

The maintenance route-planning problem (MRPP) discussed in this paper is a version of a multiple TSP with profits, in some cases named as a multivehicle routing problem with profits (Feillet et al. 2005). MRPP has similarities also to a team orienteering problem (Chao et al. 1996a). However, we derive cost functions and constraints from a different point of view, and therefore the problem has a unique nature. The main differences are time-limited routes and multi-objective nature, which are included in MRPP. We provide solutions MRPP problem using heuristic and ant colony optimization (ACO) algorithms. The heuristic algorithm has similarities to the heuristic solution to the team orienteering problem presented in Chao et al. (1996b). Other solving algorithms are based on evolutionary computing methods. ACO is considered based on the vehicle routing approach (see Bell and McMullen 2004 and Yu et al. 2009). Additionally, a genetic algorithm (GA) for MRPP based on the ordinary GA solutions for TSP is presented in Hasu and Koivo (2009). Since we have found that ACO is more efficient for MRPP, we do focus on this solution in Section 3. 


\section{From quality information to the maintenance variables}

Quality assurance systems process huge amounts of data and form information on the state of the process, the quality of which is under interest. From the maintenance perspective, the first issue to consider is how large the maintenance need of a sensor or actuator is. The reliability is considered through other concepts. In measurement networks, we can consider the reliability through availability and accuracy of observations. Availability refers to the ratio of available observations and the expected number of observations. Accuracy refers to the estimated accuracy of the measurement. Reliability is the combined effect of the availability and accuracy. That is, if either availability or accuracy is compromised, the measurement is not reliable. In probabilistic terms, the reliability 1 can be seen as the intersection of the availability and accuracy. That is, a completely reliable measurement is both available and accurate all the time. All described concepts are measured by indices varying from 0 and 1 with larger values corresponding to the better performance.

Hölttä (2009) has stated requirements for the plant performance indices, which are applicable for the maintenance variables in this chapter. First, no additional measurements should be required for the determination of indices. Second, setting up the indices should happen automatically. Third, the indices should be easy to understand. Fourth, the high level information should indicate the user easily towards the problematic point. The above concepts of reliability, availability and accuracy suit the demands very well.

The maintenance strategy of a sensor and actuator network should always be made according to the needs of the network. That is, the acceptable inaccuracy in operation should not affect the performance indices. In the end, the operational condition depends on the defined reliability, and therefore the reliability concept should include all of the essential elements to be reflected in the maintenance and nothing else.

The linguistic concepts depend on the mathematical definitions, which must be made according to the case. For example, different measurement networks have different requirements in spatial and temporal resolutions, as well as the measured fields have different time constants and spatial dependencies. For the spatial resolution, one factor influencing the mathematical definitions of performance indices is how acceptable is the estimation of missing measurements based on the neighbor measurement stations. A related issue is the ability to estimate the missing measurements. This could be rephrased as the ratio between the average station spacing and the desired spatial accuracy. In the temporal resolution, the essential issue is the difference between the system's time constant defining the dynamical behavior of the system and the sampling interval. This is related to the question of criticality of occasional missing measurement values.

\subsection{Performance indices for sensor networks}

The maintenance of a measurement network must be considered through the need for maintenance in each measurement. In here, the measurement reliability is considered through two other concepts: availability and accuracy. Availability refers to the ratio of measurements being available. Accuracy refers to the estimated accuracy of the measurement. The essence of this subsection is presented in Hasu and Koivo (2007). 
Availability $s_{i}$ expresses the availability ratio of the measurement $i$. If the measurement $i$ has not ever been missing from the data bank, $s_{i}=1$. If the measurement $i$ has always been missing, $s_{i}=0$. The measurement might be missing, for example, due to communication or measurement device errors.

The accuracy $t_{i}$ describes the estimated accuracy of the measurement $i$. The accuracy takes into account all known defects in the measurement accuracy. An accurate measurement has $t_{i}$ equal to one and a definitely inaccurate measurement has $t_{i}$ close to zero. Hence the input of the accuracy can be e.g. decision variables of the fault detection. As in the availability, the accuracy should put emphasis on the most recent events. Additionally, if different types of errors, e.g. noise or drift, can be detected, all of these should be integrated into $t$.

Reliability is the combined effect of the availability and accuracy. That is, if either availability or accuracy is compromised, the measurement is not reliable. In probabilistic terms the reliability $l$ can be seen as the intersection of the availability and accuracy. That is, a completely reliable measurement is both available and accurate all the time.

Since the measurements might run for long periods, it is advisable to use recursive techniques in order to have more emphasis on more recent events and reduce the computational requirements. Basically, this recursion increases and decreases the availability depending whether or not the measurement is available.

In Hasu and Koivo (2007), the availability was considered through the recursion

$$
s_{i}(k)= \begin{cases}\lambda \cdot s_{i}(k-1)+(1-\lambda), & \text { if } x_{i}(k) \notin M, \\ \lambda \cdot s_{i}(k-1), & \text { if } x_{i}(k) \in M \text { and } \exists j \in S \text { s.t. } x_{j}(k) \notin M, \\ s_{i}(k-1), & \text { if } x_{j}(k) \in M \forall j \in S,\end{cases}
$$

where $\lambda$ is the forgetting factor of recursive methods, $\lambda \in(0,1), M$ is the set of missing observations and $S$ is the set of measurement stations in the system. In here $\lambda$ serves also as a penalization factor - (1) shows that smaller $\lambda$ causes a larger decrease in $s$ when the measurement is missing - and larger increase if the measurement is available. Hence the small forgetting factor results in a smaller dependency to $s$ further back in history. Note also that (1) does not penalize a single measurement station if all simultaneous observations are missing in the system. This is since these types of problems often refer to communication or database problems, which are not related to measurement stations.

The update on the accuracy index can depend very much on application. The simplest way is to utilize the existing quality control tests for the observation in similar fashion to (1). That is, the accuracy can be updated through a recursion

$$
t_{i}(k)= \begin{cases}\mu \cdot t_{i}(k-1)+(1-\mu), & \text { if } x_{i}(k) \notin E, \\ \mu \cdot t_{i}(k-1), & \text { if } x_{i}(k) \in E,\end{cases}
$$

where $\mu$ is the forgetting factor for accuracy, $\mu \in(0,1)$, and $E$ is the set of erroneous observations. 
However, if the applied quality assurance techniques follow the measurement quality closer, their statistics can be utilized in the accuracy computation. Hasu and Koivo (2007) present an example, in which the filter residual $r_{i}(k)$ and its respective standard deviation $\sigma_{i, r}$ update the accuracy. Mathematically this is essentially represented by

$$
t_{i}(k)=\left(\frac{\lambda_{1}}{2}+\frac{\lambda_{2}}{2}\right) \cdot t_{i}(k-1)+\left(1-\frac{\lambda_{1}}{2}\right)\left(1-f\left(r_{i}(k), \sigma_{i}(k)\right)+\left(1-\frac{\lambda_{2}}{2}\right)\left(1-g\left(\sigma_{i}(k), \sigma_{i}^{\min }(k)\right),\right.\right.
$$

where $\lambda_{1}$ and $\lambda_{2}$ are forgetting factors for relative and absolute inaccuracies of the measurement, and

$$
\begin{gathered}
f\left(r_{i}(k), \sigma_{i}(k)\right)=\left\{\begin{array}{cc}
0, & r_{i}(k)<3 \sigma_{i}(k), \\
2 \cdot P\left(r_{i}(k) / \sigma_{i}(k)-4\right), & r_{i}(k) \geq 3 \sigma_{i}(k),
\end{array}\right. \\
g\left(\sigma_{i}(k), \sigma_{i}^{\min }(k)\right)=P\left(3 \sigma_{i}(k) / \sigma_{i}^{\min }(k)-9\right), \\
P(x)=\left(1+e^{-x}\right)^{-1},
\end{gathered}
$$

where $\sigma_{i}^{\min }$ is the minimum residual standard deviation in the measurement network, and $P$ is the sigmoid function. Even though the update (3)-(6) looks a bit complicated, it includes only simple computations with variables.

As mentioned, the reliability is considered as the intersection of availability and accuracy. Hence, as in probability theory, we define the reliability as the product of these two:

$$
l_{i}(k)=s_{i}(k) t_{i}(k) .
$$

Note that this definition enables the easy use of additional performance indices as the availability and accuracy in the determination of reliability. For example, other accuracy variables can be included in the form of $t=t^{1} t^{2} \ldots t^{n}$, where the superscripts indicate the numbers of accuracy subindices. In (7), we could use a variety of methods to integrate different performance indices into higher level indices (see Hölttä 2009 or Hasu and Koivo 2009). The other possibilities include taking minimum, maximum, arithmetic mean, harmonic mean or any other type of central tendency measure.

Hasu and Koivo (2007) present a numerical example of the performance indices for a weather sensor network. Figure 1 shows the availability and accuracy indices measured using the updates (1) and (3), respectively. Based on Figure 1, three stations can be described as follows. Kaukas station temperature measurement has poor availability and accuracy. Luhtaanmäki and Huhtionmäki stations suffer from a compromised temperature observation quality, and Luhtaanmäki has also occasional missing observations. The corresponding reliability values from these stations are drawn in Figure 2, where also a reference station of Kantele is included.

Figure 3 shows the observations from a reference station of Kantele, which does not suffer from any problems, as well as the Kaukas and Huhtionmäki stations presented in Figure 1. Comparing the Huhtionmäki observations with the Kantele observations shows how the 
Kantele measurement suffers from noise in the five-minute sampling, and therefore the accuracy is compromised. Figure 3 shows that problems of Kaukas Station relate to a massive number of missing observations and large noise in the daytime observations.
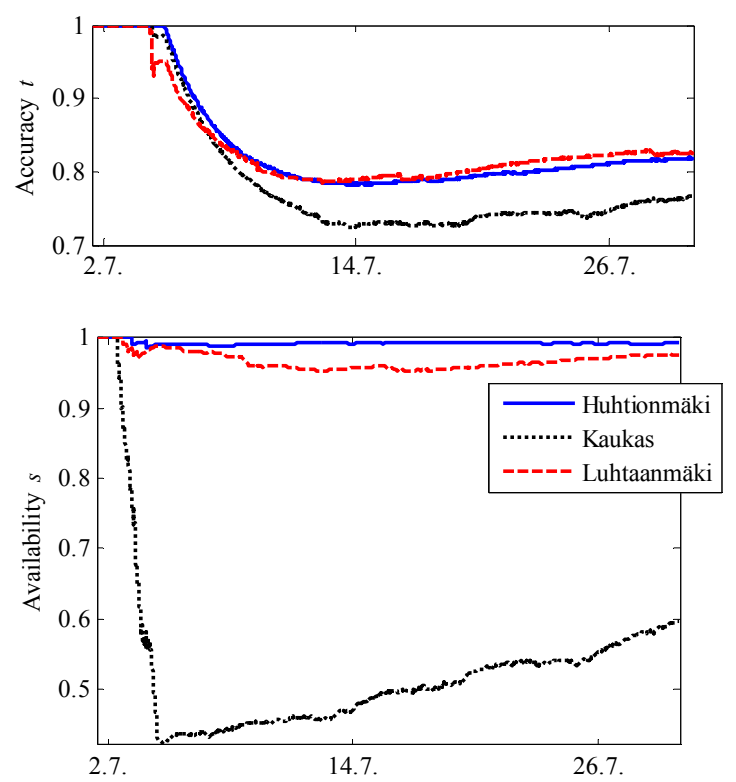

Fig. 1. Availability s and accuracy $t$ from three temperature measurement stations in the Southern Finland.

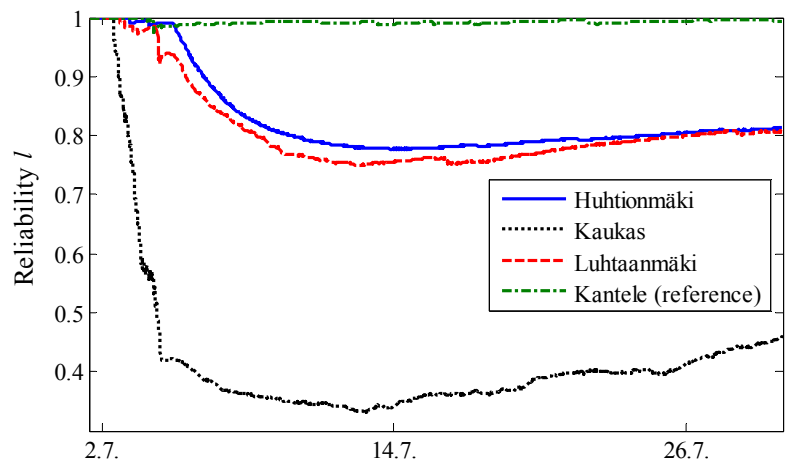

Fig. 2. The reliabilities of the above stations with a reference station Kantele.

In overall, the above numerical example exemplifies how the measurement quality can be described easily using a few index. First, the reliability index shows the overall quality. Second, the accuracy and availability describe the sources of potential problems. If desired, the hierarchy on the accuracy side could be further constructed to identify the nature of the problems - thus helping to choose the correct types of maintenance actions. 

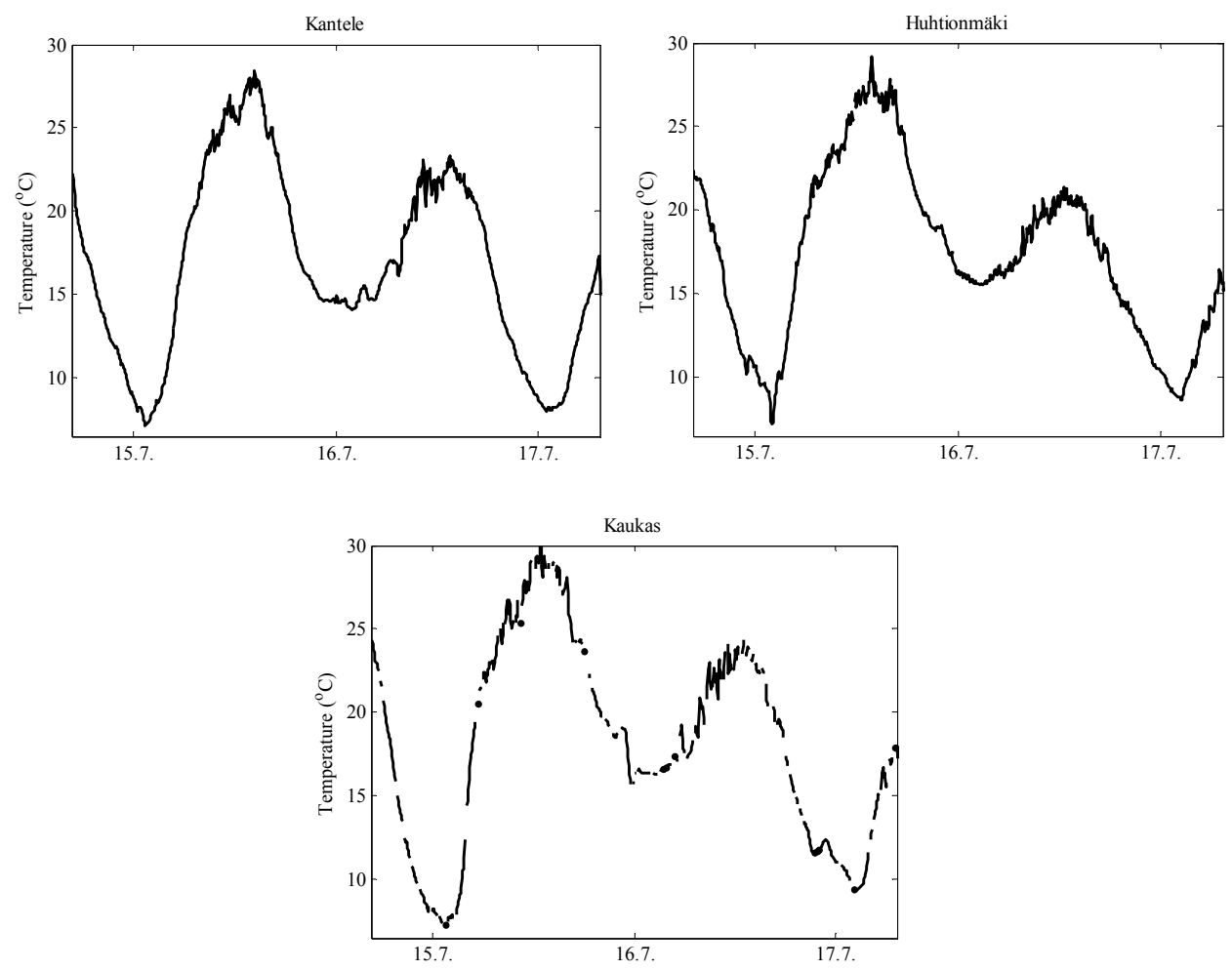

Fig. 3. Observations from Kantele (top left), Huhtionmäki (top right) and Kaukas (bottom) stations.

\subsection{Performance indices for actuator networks}

In the actuator networks, the actuator performance relates to the control performance of the (sub-)system. Hölttä and Koivo (2011) presents several performance indices for the determination of the control performance. Some of the performance indices are more application-related but in the following, we have collected a few generic performance indices for actuator performance.

The first actuator performance index, the duration of setpoint error, originates from the work of Jämsä-Jounela et al. (2003). This index follows the control loop performance by detecting the time of permanent error. That is, this index indicates if the control loop suffers from a permanent error. The duration index is updated using

$$
I(k)=\lambda I(k-1)+(1-\lambda) \cdot\left\{\begin{array}{l}
\operatorname{sgn}(e), \text { if }|e|>e_{\lim } \\
0, \text { if }|e| \leq e_{\lim },
\end{array}\right.
$$

where $e$ is the tracking error between the setpoint and system output, $e_{\text {lim }}$ represents the boundary of the acceptable region and sgn stands for the signum function. Since our goal is 
to have positive indices, for which zero and one stand for poor and good performance, repectively, and we are not interested in the sign of the setpoint error, the final index is

$$
\eta(k)=P\left(\frac{k}{25}\left(\eta_{50}-|I(k)|\right)\right) \text {. }
$$

That is, the absolute value of $I$ is scaled using the sigmoid function (6) using constants $k$ and $\eta_{50}$, which describe respectively the scale and location (midpoint) of the sigmoid. The scaling constants depend on the application. Additionally, Hölttä and Koivo (2011) present a variation of (8) for the magnitude of the set point error, using essentially the following

$$
I(k)=\lambda I(k-1)+(1-\lambda) \cdot\left\{\begin{array}{l}
\operatorname{sgn}(e)\left(\operatorname{sgn}(e) e-e_{\lim }\right)^{2}, \text { if }|e|>e_{\lim }, \\
0, \text { if }|e| \leq e_{\lim } .
\end{array}\right.
$$

Again, the final index is obtained using (9). Obviously, this index describes the temporally weighted average for the setpoint error.

More detailed control performance indices can be formed. Hölttä (2009) presents a version of sluggish control index originally introduced by Hägglund (1999). This index grows if the control loop follows the control signal slowly. The index is defined as

$$
I(k)=\lambda I(k-1)+(1-\lambda) \cdot \operatorname{sgn}(\Delta u \Delta y),
$$

where $\Delta u$ and $\Delta y$ are the control and output increments, respectively. The values close to one indicate a sluggish control loop. Due to possible negative values, Hölttä uses the scaling similar to (9) in order to form the final performance index.

A numeric simulation exemplifies the strength of this approach. We simulate a system described in Figure 4, including a plant with transfer function $1 /(\mathrm{s} 2+2 \mathrm{~s}+2)$ and a PIcontroller. The pulse-like reference is drawn as the black line in Figure 5. The controller is applied with two tunings, from which the first one $\left(K=2, T_{i}=2 / 3\right)$ is much slower than the second one $\left(K=1 / 2, T_{i}=1 / 2\right)$, to which the controller is switched at $t=50$.

The system output is plotted in blue in the top of Figure 5. The first tuning reaches the reference slowly compared with the second tuning. This is visible also in the performance index for sluggish control, which is included in the bottom of Figure 5. The permanent error index behaves similarly. It, however, does not reach as low values due to the change of reference. The performance indices are combined into a loop index (the black dotted line at the bottom of Figure 5) using multiplication.

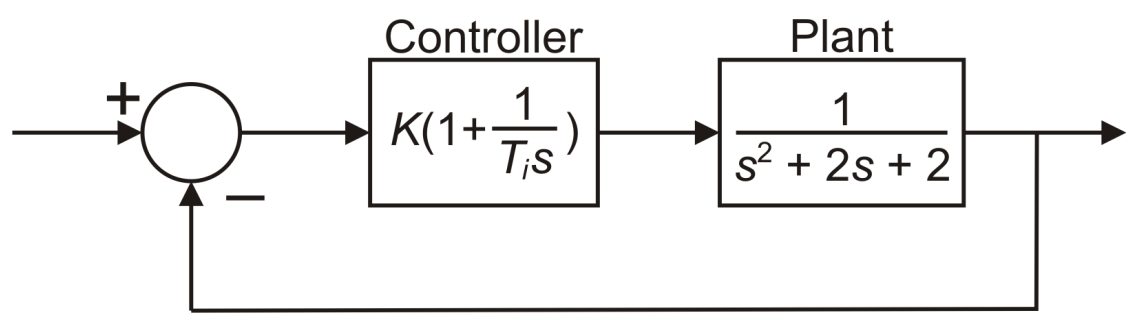

Fig. 4. The simulated control loop. 

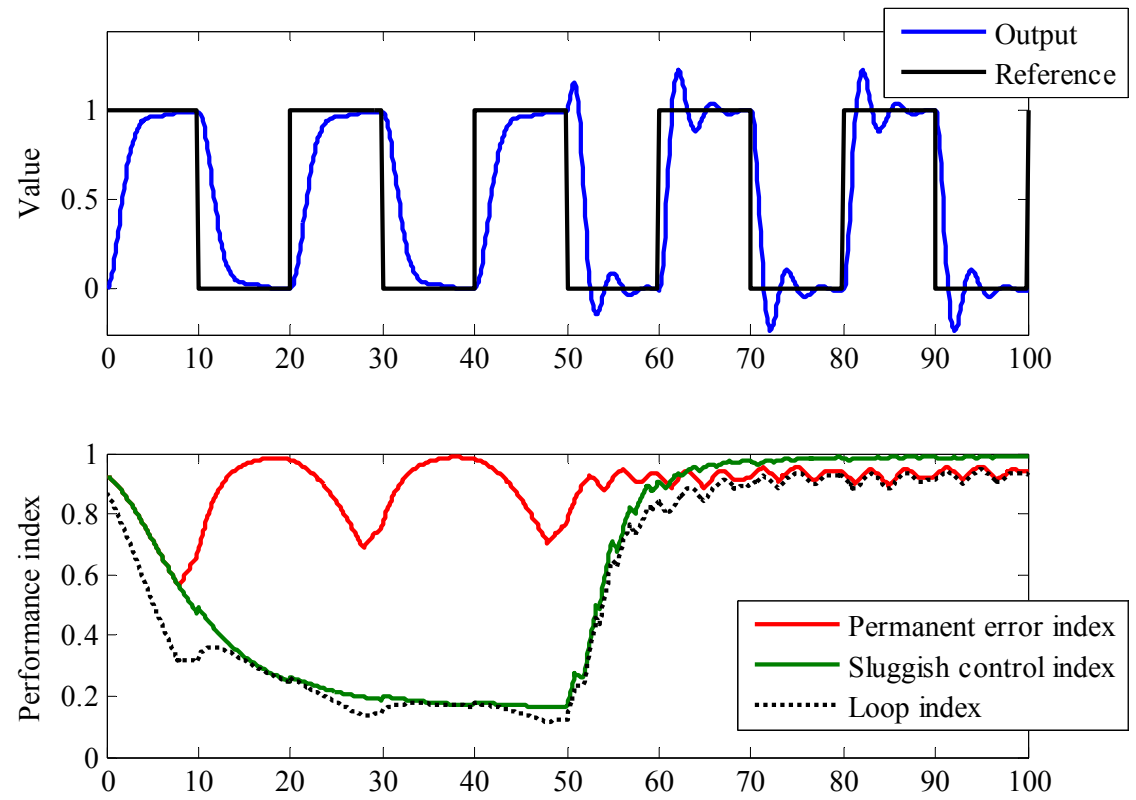

Fig. 5. The simulated output (top) and performance indices (bottom).

\subsection{Performance Indices using fuzzy inference}

In addition to the recursive techniques, the performance indices can be calculated using other methods - as well as the requirements for the indices mentioned at the beginning of Section 2 are met. One interesting option is to use fuzzy inference. Hasu and Koivo (2009) have presented one version how to apply fuzzy inference for the measurement accuracy and availability performance indices.

The idea of the fuzzy system is to replace the equations (1)-(6), which are applied in the index computation. Hence the inputs to the fuzzy inference include the previous index value. The other input to the accuracy is the QA information about the erroneousness of the observation and for the availability the other input is the period from the previous observation (as a multiple of sampling time). The applied fuzzy system is based on the Mamdani inference with triangular membership functions and multiplicative intersection, implication and aggregation. The membership functions for the accuracy and availability are presented in Figure 6.

The fuzzy rules for the accuracy and availability are collected into Table 1 . The resulting fuzzy interference surfaces are included in Figure 7. Note that for the accuracy we have used only "good/erroneous observation" classification for the measurement quality - this corresponds to the accuracy index presented by (2). 

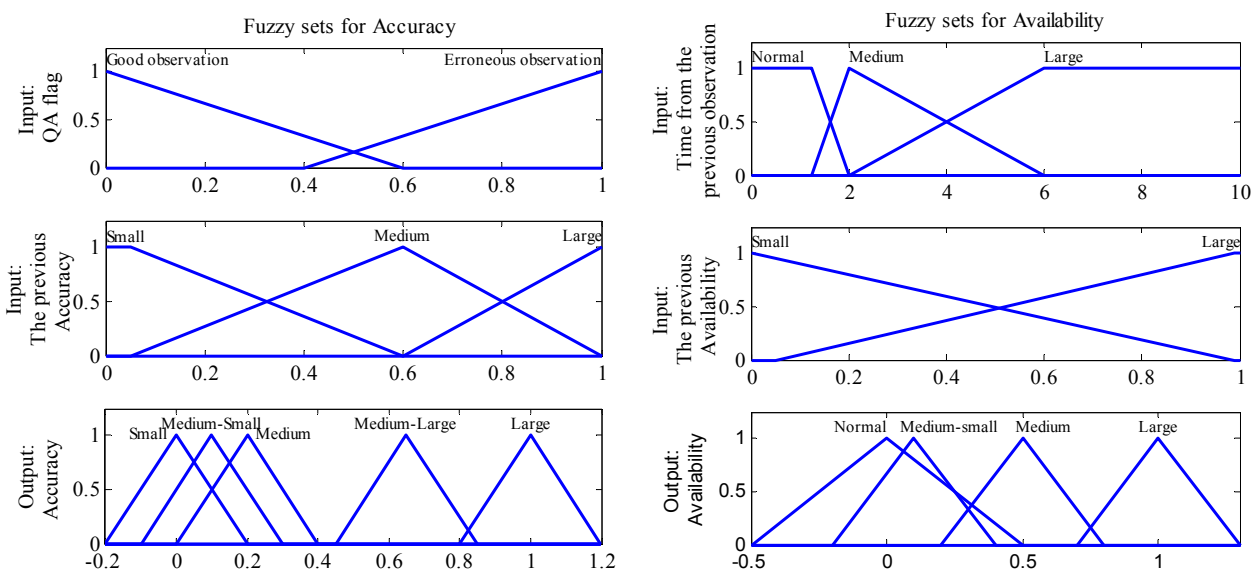

Fig. 6. The fuzzy sets for the accuracy and availability indices.

\begin{tabular}{|c|c|c|c|c|c|}
\hline \multicolumn{3}{|c|}{ Accuracy } & \multicolumn{3}{|c|}{ Availability } \\
\hline $\begin{array}{l}\text { Input: QA } \\
\text { indication for the } \\
\text { observation }\end{array}$ & $\begin{array}{l}\text { Input: The } \\
\text { previous } \\
\text { accuracy }\end{array}$ & $\begin{array}{l}\text { Output: } \\
\text { Accuracy }\end{array}$ & $\begin{array}{l}\text { Input: Time from } \\
\text { the previous } \\
\text { observation }\end{array}$ & $\begin{array}{l}\text { Input: The } \\
\text { previous } \\
\text { availability }\end{array}$ & $\begin{array}{l}\text { Output: } \\
\text { Availability }\end{array}$ \\
\hline Good & Large & Large & Normal & Large & Large \\
\hline Good & Medium & Medium-large & Normal & Small & Medium-small \\
\hline Good & Small & Medium-small & Medium & Large & Medium \\
\hline Erroneous & Large & Medium & Medium & Small & Medium-small \\
\hline Erroneous & Medium & Medium-small & Large & Large & Small \\
\hline Erroneous & Small & Small & Large & Small & Small \\
\hline
\end{tabular}

Table 1. Fuzzy inference rules.
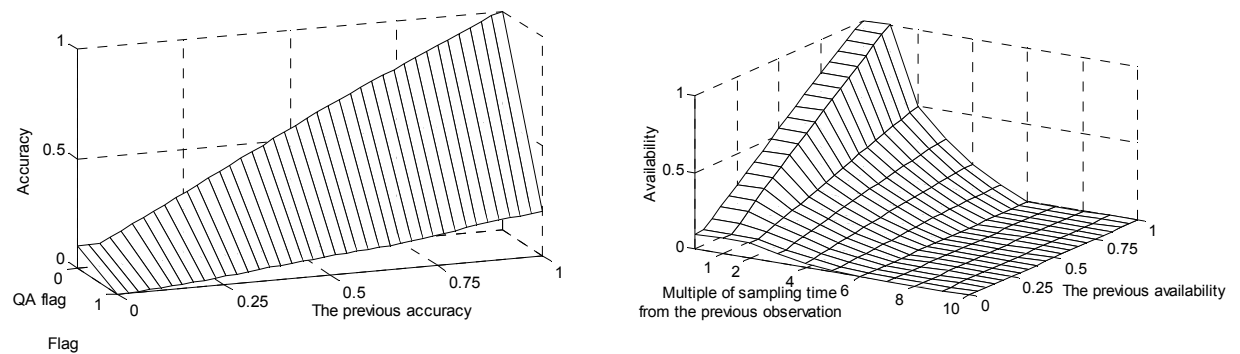

Fig. 7. The fuzzy inference surface for accuracy and availability indices. 


\section{Automatic maintenance route planning}

\subsection{Definition of maintenance route planning problem}

The maintenance route planning aims for the maximum improvement of sensor network performance with minimal costs. Since services are nowadays often outsourced, an automated tendering of subcontractors must occur in the planning stage. Planning limitations are the number of available technicians and the lengths of working days. The route planning must take into account the distances between the stations as well as the maintenance times in each station. Costs include starting, repair, and travelling costs, which may be subcontractor dependent. Additional information to take into account is the importance of stations.

MRPP is a multiobjective optimization problem. On one hand, we want to maximize the network improvement by sending technicians to stations with poor performance, i.e. small performance indices. On the other hand, we like to keep the maintenance costs as low as possible while keeping the sensor network performance still in an acceptable level. In practice, the multiobjective problem means that MRPP will have a Pareto-optimal set of optimal solutions (e.g. [7]) and therefore the choice of the best route combination is up to one's preference. In this work, we deal with the problem by presenting so-called Paretoboundary found by the algorithm.

The mathematical definition of MRPP starts from a definition of a route matrix $\mathbf{R},\left[R_{i, j}\right]_{i, j=1}^{n, m}$, where $n$ is the maximum route length plus two and $m$ is the number of available technicians. By definition, the first entry of each column corresponds to the current location, i.e. the starting point, of the technician. We use station index zero to refer to the depots of technicians. The rest of column $j$ is for the indices of stations in the order of technician $j$ 's route. Since the routes are usually shorter than the maximum route length, the column is adjoint with a suitable amount of zeros.

MRPP consists of a multiobjective function to be maximized, working day constraints and minimum gain requirements. In mathematical terms, MRPP maximization is

$$
\max _{\mathbf{R} \in \mathfrak{R}} \mathbf{f}(\mathbf{R})=\left[\sum_{j=1}^{m} \sum_{i=2}^{n-1} W\left(R_{i, j}\right)\left(1-l\left(R_{i, j}\right)\right),-\sum_{j=1}^{m} \sum_{i=2}^{n} c_{j} d\left(R_{i, j}, R_{i-1, j}\right)-\sum_{j=1}^{m} \sum_{i=2}^{n-1} C\left(R_{i, j}\right)\right],
$$

where the first entry is the network performance gain and the second entry is the opposite number of the cost. $\Re$ is the space of possible route matrices. $W\left(R_{i, j}\right)$ and $l\left(R_{i, j}\right)$ are the importance weight and reliability index, respectively. On the cost side of (12), $c_{j}$ is the hourly cost of technician $j, d\left(R_{i, j}, R_{i-1, j}\right)$ is the temporal distance between the $i$ th and the previous stations in the technician $j^{\prime}$ s route and $C\left(R_{i, j}\right)$ is the maintenance cost of station $i$ by technician $j$.

By definition, the depots do not have repair costs, i.e. $C(0)=0$, and distance from a place to itself is zero, i.e. $d(i, i)=0$. Note that (12) assumes that the starting point is already repaired, and hence solving (12) enables the updates of routes during the working day.

The working day constraint for the technician $j$ in MRPP is 


$$
\left(\sum_{i=1}^{n} d\left(R_{i, j}, R_{i-1, j}\right)+\sum_{i=2}^{n-1} t\left(R_{i, j}\right)\right) \leq T_{\max , j},
$$

where $t\left(R_{i, j}\right)$ is the maintenance time of station $i$ of technician $j$ and $T_{\max , j}$ is the length of working day of technician $j$.

Additional constraints for MRPP describe the required performance decrease of a station before maintenance is considered and the desired minimum gain from a route. These constraints remove unnecessary routes and maintenance actions, and they restrict the solution space of the optimization problem. If we define a total route gain of technician to be $G_{j}=\sum_{i=2}^{n-1} W\left(R_{i, j}\right)\left(1-l\left(R_{i, j}\right)\right)$, the minimum station gain and the minimum route gain constraints are equal to

$$
W\left(R_{i, j}\right)\left(1-l\left(R_{i, j}\right)\right) \geq g_{\min }, \forall R_{i, j} \backslash\{0\},
$$

and

$$
G_{j} \geq G_{\min } \vee G_{j}=0
$$

respectively, where $g_{\min }$ is the minimum maintenance gain of a station and $G_{\min }$ is the minimum acceptable route gain.

\subsection{Solving MRPP: A heuristic solution}

The following heuristic solution of MRPP is a greedy type algorithm introduced originally in Hasu and Koivo (2009). The algorithm adds to routes a station, which maintenance improves the network performance the most in respect to the additional cost. This is measured with a ratio between the increased network performance and the increased cost while keeping constraints (13) and (14) in mind. After the addition of a station, the heuristic checks possible station swaps between routes in order to reduce the cost described in the second entry of (12). The station swaps from one route to another are somewhat similar to orienteering problem heuristic in Chao et al. (1996b).

The ratio of the network performance increase and cost is

$$
\frac{W(k)(1-l(k))}{\min _{i, j}\left(c_{j} \Delta t(i, j)+C(k)\right)}
$$

where $\Delta t(i, j)$ refers to the temporal increase of technician $j$ 's route if station $k$ is added into $i$ th station in that route. The time addition $\Delta t$ includes both travelling and maintenance time. In here, "available" refers to a station, the addition of which satisfies (2), (3), and which is not already included in routes.

The heuristic algorithm for a subset of technicians follows the outlines described in Table 2. The route additions are greedy and the station modifications between technicians make sure that route costs do not grow unnecessarily large. 
In order to make the tendering of subcontractors and to choose the best number of technicians, the heuristic algorithm determines the routes for all possible subsets of technicians and chooses the most advantageous route ensemble. For example, if two subcontractors with one technician are available for the maintenance, the heuristic determines separate routes for each of them separately (i.e. $\mathbf{R}$ is a column vector) and one route combination with both technicians (i.e. $\mathbf{R}$ is a two-column matrix). The algorithm chooses the route matrix, which maximizes the first entry of $f$ in (12). If a route does not satisfy (15), the algorithm discards the whole solution in the corresponding route matrix.

WHILE $\exists$ station index $\mathrm{k} \notin \mathrm{R}$

FIND the maximum ratio (16) over $\mathrm{k} \notin \mathrm{R}$ satisfying (13) and (14), and over $\Delta \mathrm{t}(\mathrm{i}, \mathrm{j})$ and ADD index $\mathrm{k}$ into ith slot of the route of technician $\mathrm{j}$

IF $\nexists$ k satisfying the above conditions

END IF

END WHILE

IF moving any station from technician $\mathrm{j}$ to other technicians would reduce the total costs MOVE the selected station from technician $j$ to an another technician

END IF

IF swapping a station from technician $\mathrm{j}$ to any other technician's station would reduce the costs END IF SWAP the selected stations

END WHILE

Table 2. Heuristic maintenance route determination.

\subsection{Solving MRPP: Ant colony optimization}

ACO is a widely used method for solving combinatorial optimization problems, to which group includes also MRPP. ACO is inspired by the way ant colonies find their food in nature. Basically, ants leave pheromone to mark good routes to find food and the following ants are likely to choose the route parts with pheromone. In ACO, the routes ranked as good solutions to the problem receive pheromone, and therefore those parts are more likely used also in the future.

As mentioned in Section 1, MRPP has similarities to the vehicle routing problem and therefore our ACO algorithm for MRPP is based on a few vehicle routing algorithms. The biobjectivity of the gain function $\mathbf{f}$ defined in (12) is taken care of using a similar approach to Schilde et al. (2009), where two different pheromones are used for the different objectives. That is, the pheromone additions are done with the similar all-for-the-few-best fashion. We collect the Pareto-optimal solutions to so-called Pareto-optimal set, in which no solution dominates completely other (i.e. none of the solutions is better than other in respect to the both gain function entries). The multiple starting depots of technicians are handled as in $\mathrm{Yu}$ et al. (2011): the first and last locations in routes are set to each technician's depot.

In ACO for MRPP, an ant population of $q$ groups of $m$ ants ( $m$ is the number of technicians) wander through to the possible destinations without violating the MRPP constraints (13) and (14) for each group. That is, during the route construction phase, $m$ ants start from their respective depots in random order to build route $\mathbf{R}_{n}, n=1, \ldots, q$, to a random station one station a time. 
The main algorithm for ACO solving MRPP is presented in Table 3. In the following, we describe the algorithm steps more closely.

COMPUTE heuristic information matrices $\eta_{j i k 1}$ and $\eta_{j i k 2}$.

INITIALIZE the pheromone matrices $\tau_{j i k 1}$ and $\tau_{j i k 2}$.

WHILE iteration stopping condition is not met

CREATE $q$ groups of $m$ ants with randomized starting order if there are more than one depots.

FOR $q$ groups

WHILE $\exists$ stations that are feasible to add to current group, i.e. $\Omega(\mathbf{R}) \neq \varnothing$.

FOR ant 1 to ant $m$.

SELECT randomly a new station to add to the route using weighting based on the pheromone and heuristic information.

END FOR

UPDATE the pheromones using the evaporation: $\tau_{j i k a} \leftarrow(1-\rho) \tau_{j i k a}+\rho \tau_{0}$.

\section{END WHILE}

APPLY improvement strategies: route permutation, switching and removing stations, and the replacing a route from the Pareto-solutions.

END FOR

UPDATE the pheromones with the global update according to the best route combinations based on the separate gain functions and the equally weighted gain.

UPDATE the set of Pareto-solutions.

END WHILE

Table 3. ACO maintenance route determination.

Before ACO algorithm starts to increase route lengths, the heuristic information and initial pheromone levels must be determined. The heuristic information is applied in the route formation for the transition probabilities from one station to another - representing the natural attractiveness of ant $j$ moving from the current station $i$ to the station $k$. This attractiveness depends on the gain function, which is maximized. Since we are dealing with a bi-objective optimization (12), we have separate heuristic information for both of the goals of $\mathbf{f}$. The heuristic information are calculated using

$$
\eta_{j i k 1}=\frac{1}{c_{j} \Delta t(i, j)+C(k)}, \eta_{j i k 2}=W(k)(1-l(k)) .
$$

The first heuristic information is the inverse of the additional cost and the second is related to the gain corresponding to maintaining the station $k$. Note how these compare with the numerator and denominator of the heuristic method's decision variable (16). In addition to the heuristic information, the ACO pheromone levels must be initialized. Initially, the pheromones of technician $j$ moving from station $i$ to station $k$ in respect to the objective $a$ are set to value $\tau_{0}$, i.e. $\tau_{j i k a}=\tau_{0}$.

In here, the next random station is chosen from the set of stations satisfying constraints (13) and (14) with probabilities given by

$$
p\left(v_{j i k}\right)= \begin{cases}\frac{\tau_{j i k 1} \eta_{j i k 1}^{2} p_{1}+\tau_{j i k 2} \eta_{j i k 2}^{2} p_{2}}{\sum_{h \in \Omega(x)}\left(\tau_{j i h 1} \eta_{j i h 1}^{2} p_{1}+\tau_{j i h 2} \eta_{j i h 2}^{2} p_{2}\right)}, & \text { if } h \in \Omega(\mathbf{R}), \\ 0, & \text { otherwise }\end{cases}
$$


where $\Omega(\mathbf{R})$ is the index set of stations, which are available for a visit i.e. not visited by any of the $m$ ants (technicians) and addition of these stations does not violate (13) or (14), and $p_{a}$ is the weight of ath goal in (12) for the current group of $m$ ants. If the station is added to a route of any ant in grouped in $\mathbf{R}$, the station is removed from $\Omega(\mathbf{R})$.

Every time a new station $k$ is added to route $\mathbf{R}$, its pheromone is evaporated in order to reach the improved diversification of solutions. This means that for other groups of ants, unvisited stations become more attractive. The pheromone evaporation is done by

$$
\tau_{j i k a} \leftarrow(1-\rho) \tau_{j i k a}+\rho \tau_{0},
$$

where $\rho$ is a forgetting factor called evaporation constant and $a$ is 1 or 2 . Since the attractiveness of moving between stations $i$ and $k$ does not depend on the direction of movement, we make also an additional update $\tau_{j k i a}=\tau_{j i k a}$ for symmetry.

After a group of $m$ ants has finalized its routes, i.e. no more stations can be added to routes, route improvement strategies are applied to reach better solutions. If the strategies improve the solution at least in Pareto sense (i.e. value at least one objective of $\mathbf{f}$ is improved), the solution is added to the population having originally $q$ solutions. Literature knows a variety of such strategies (e.g. Yu et al. 2011, Schilde et al. 2009, Bell and McMullen 2004).

In our solution, we use three techniques, from which two first ones are based on the literature (e.g. Schilde et al. 2009) and the last one is developed especially for this problem. First, we permutate the stations in the route of each technician to find if the stations can be arranged to lower cost order. If the route has many stations, we limit the permutations to 50. Second, for a fraction of groups, we either switch one station from route to another or completely remove one station from the route. The first option tries to minimize the total costs and the second option finds possible Pareto-optimal solutions. Third, we replace a single technician's route with a route from the set of Pareto-optimal solutions and see if the new route combination is better at least in Pareto sense. This way we increase the number of route combinations using known good routes and improve the Pareto set of solutions.

In order to emphasize the good solutions, after each round of making all $q$ sets of routes, we make a global update for the pheromones. The updates are made as in Schilde et al. (2009); the pheromones of the best solution in respect to one of the entries in gain function (12) is increased using $\tau_{j i k a} \leftarrow \tau_{j i k a}+\tau_{0}$, and for the second best solution the update is $\tau_{j i k a} \leftarrow \tau_{j i k a}+$ $\tau_{0} / 2$, where $a$ is the number of the gain function entry.

In addition to emphasize the multi-objectivity, we make also a compromise update between the gain function entries for the routes, which reach the maximum value when both gain function entries are weighted equally. These pheromone updates are done by $\tau_{j i k a} \leftarrow \tau_{j i k a}+$ $\tau_{0} / 2$, and for the second best solution the update is $\tau_{j i k a} \leftarrow \tau_{j i k a}+\tau_{0} / 4$, where $a$ is 1 and 2 . Also for the global update, we do the symmetry update $\tau_{j k i a}=\tau_{j i k a}$.

After each round, the solutions are compared with the Pareto-optimal set of solutions. In the Pareto-optimal set is included all routes, which do not have completely dominating solutions, i.e. no other solution has better values in both entries of the gain function $\mathbf{f}$. 


\subsection{Maintenance routing examples}

The following simulated case examples demonstrate the maintenance routing. In all simulations, the working day length is limited to 7.5 hours, and the travel times between stations are calculated based on $60 \mathrm{~km} / \mathrm{h}$ speed. The MRPP parameters $G_{\min }$ and $g_{\min }$ are set to 2 and 0.5 , respectively. In all cases, the heuristic algorithm routes are included to the first iteration round of ACO as an initialization help for the pheromone. The maintenance takes 0.5 hours per station in the smaller examples and 1 hour per station in the large example. In our examples we use the initial ACO pheromone value $\tau_{0}=1$.

Figure 8 shows a small simulated example of maintenance route formation, in which the routes of heuristic and ACO algorithms are included. The ACO algorithm was used with 75 ant groups for 50 iterations. In the left part of Figure 8, we have one technician starting from the depot (black diamond) and in the right part we have two technicians starting from different depots. The heuristic algorithm routes reach the total gains of 8.9 and 13.2 and the total costs of 12.5 and 24.0, whereas the ACO algorithm routes have the total gains 8.9 and 13.5 and the total costs 12.4 and 23.1. The ACO routes are chosen from the Pareto set of solutions based on the closeness to the heuristic solution.
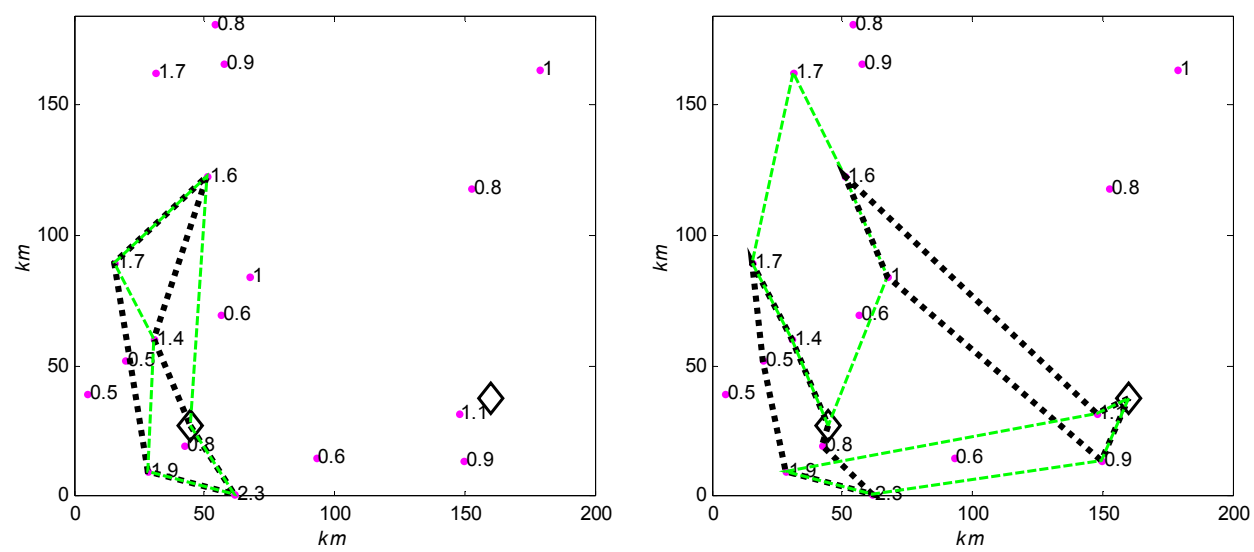

Fig. 8. Maintenance route examples for two depots. Magenta dots are the stations to be maintained and the numbers next to them present the maintenance gain available. Black dotted lines present the heuristic algorithm routes and the green dashed lines are the ACO routes.

Figure 9 shows an example with larger number of maintenance technicians and stations requiring maintenance. In here, two depots have both two technicians and one depot has a technician for the maintenance of the network. The ACO algorithm is run with 100 ant groups of five for 50 iterations.

For the routes in Figure 9, the heuristic algorithm routes the total cost and gain are 53.9 and 27.0, respectively, whereas the presented ACO route solution has the cost of 53.4 and the gain of 28.0. 
The experiences on these types of large problems with many technicians having relatively short routes is that the improvement strategy of picking good routes from the Paretooptimal solutions is very important in improving the overall optimization results.

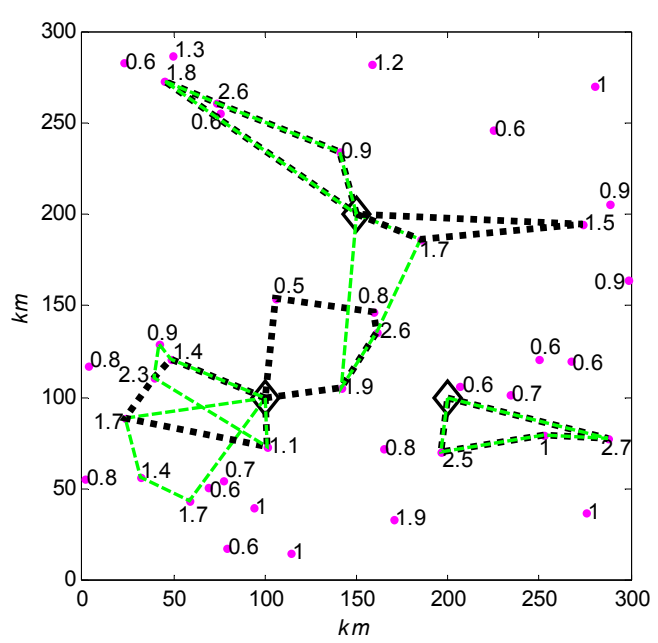

Fig. 9. A large maintenance routes example with three depots and five technicians. Magenta dots are the stations to be maintained and the numbers next to them present the maintenance gain available. Black dotted lines present the heuristic algorithm routes and the green dashed lines are the ACO routes.

\section{Final remarks}

The maintenance is an essential part in the continuum of quality assurance. Automated maintenance planning can offer a valuable tool for extensive and widely distributed networks, for which the maintenance managing would otherwise be tedious. The approach presented in here has three main steps. First, the performance indices are extracted from the essential QA information. As demonstrated in Section 2, the indices are applicable in sensor and actuator networks. Second, the performance indices are transformed into gains, which measure how much maintenance actions can improve the network performance. Third, the maintenance routes are solved using the methods in Section 3.

Solving the MRPP presented as in Section 3 offers several properties needed in practical systems; such as the optimization of network performance with given resources, tendering the outsourced maintenance services on case-by-case-basis, and the ability to update the maintenance strategy if the situation has changed in the network. The multiobjective nature of the problem leads to the Pareto-optimal set of solutions and the best solution must be determined depending on the relation between the improved performance and costs in the network.

\section{Acknowledgments}

This work was funded by the Finnish Fund for Automation, Finnish Funding Agency for Technology and Innovation (Tekes) and Vaisala Oyj. 


\section{References}

J.E. Bell, and P.R. McMullen, 2004: "Ant colony optimization techniques for the vehicle routing problem", Ad. Eng. Informatics, vol. 18, no. 1, pp. 41-48.

I.M. Chao, B.L. Golden, and E.A. Wasil, 1996a: “The Team Orienteering Problem”, Europ. J. Operational Res., vol. 88, no. 3, pp. 464-474.

I.M. Chao, B.L. Golden, and E.A. Wasil, 1996b: "A Fast and Effective Heuristic for the Team Orienteering Problem”, Europ. J. Operational Res., vol. 88, no. 3, pp. 475-489.

D. Feillet, P. Dejax, and M. Gendreau, 2005: “Traveling Salesman Problems with Profits: An Overview", Transportation Science, vol. 39, no. 2, pp. 188-205.

T. Hägglund, 1999: "Automatic detection of sluggish control loops", Control Engineering Practice, vol. 7, no. 12, pp. 1505-1511.

V. Hasu, and H. Koivo, 2007: "Maintenance variables for spatially distributed measurement networks," Proc. IEEE Int. Conf. Systems, Man, and Cybernetics, Montreal, Canada, IEEE, pp. 1327-1332.

V. Hasu, and H. Koivo, 2009: "Automatic maintenance route planning of large-scale sensor networks," Proc. IEEE Int. Conf. Computat. Intellig. for Meas. Syst. and Appl., Hong Kong, China, IEEE, pp. 18-23.

V. Hölttä, 2009: "Plant Performance Evaluation in Complex Industrial Applications", Doctoral Thesis, Helsinki University of Technology, $123 \mathrm{p}$.

V. Hölttä, and H. Koivo, 2009: “Quality index framework for plant-wide performance evaluation", J. Process Control, vol. 19, no. 7, pp. 1143-1148.

V. Hölttä, and H. Koivo, 2011: "Performance metrics for web-forming processes", J. Process Control, vol. 21, pp. 885-892.

R. Isermann, 2006: “Fault-Diagnosis Systems - An Introduction from Fault Detection to Fault Tolerance", Springer-Verlag.

S.-L. Jämsä-Jounela, R. Poikonen, N. Vatanski, A. Rantala, 2003: “Evaluation of control performance: methods, monitoring tool and applications in a flotation plant", Minerals Engineering, vol. 16, pp. 1069-1074.

C.M.H. Kuijpers, R.H. Murga, I. Inza, and S. Dizdarevic, 1999 “Genetic algorithms for the travelling salesman problem: A review of representations and operators", Artificial Intelligence Review, vol. 13, no. 2, pp. 129-170.

M. Schilde, K.F. Doerner, R.F. Hartl, G. Kiechle, 2009: "Metaheuristics for the bi-objective orienteering problem", Swarm Intell., vol. 3, no. 3, pp. 179-201.

J.C.S. de Souza, M.B. Do Coutto Filho, M.T. Schilling, C. de Capdeville, 2005: “Optimal Metering Systems for Monitoring Power Networks under Multiple Topological Scenarios", IEEE Trans. Power Systems, vol. 20, no. 4., pp. 1700-1708.

T. Sun, L.-J. Chen, C.-C. Han, and M. Gerla, 2005: "Reliable Sensor Networks for Planet Exploration", Proc. IEEE Conf. on Networking, Sensing, and Control ICNSC 2005, pp. 816-821.

N. Wang, J.-C. Lu, and P. Kvam, 2006: “Reliability Modeling in Spatially Distributed Logistics Systems", IEEE Trans. Reliability, vol. 55, no. 3, pp. 525-534.

B. Yu, Z.-Z. Yang, and B. Yao, 2009: “An improved ant colony optimization for vehicle routing problem", Eur. J. Operat. Res., vol. 196, no 1, pp. 171-176.

B. Yu, Z.-Z. Yang, and J.-X. Xie, 2011: "A parallel improved ant colony optimization for multi-depot vehicle routing problem", J. Operational Research Society, vol. 62, no. 1, pp. 183-188. 


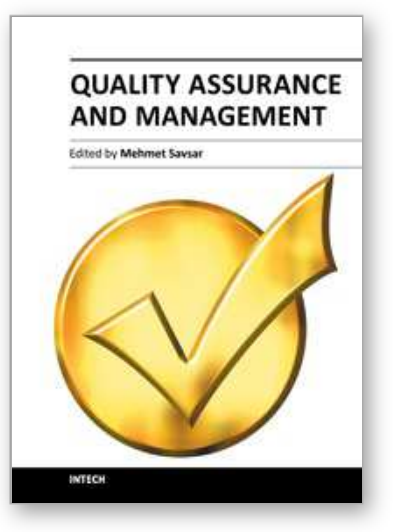

\author{
Quality Assurance and Management \\ Edited by Prof. Mehmet Savsar
}

ISBN 978-953-51-0378-3

Hard cover, 424 pages

Publisher InTech

Published online 23, March, 2012

Published in print edition March, 2012

The purpose of this book is to present new concepts, state-of-the-art techniques and advances in quality related research. Novel ideas and current developments in the field of quality assurance and related topics are presented in different chapters, which are organized according to application areas. Initial chapters present basic ideas and historical perspectives on quality, while subsequent chapters present quality assurance applications in education, healthcare, medicine, software development, service industry, and other technical areas. This book is a valuable contribution to the literature in the field of quality assurance and quality management. The primary target audience for the book includes students, researchers, quality engineers, production and process managers, and professionals who are interested in quality assurance and related areas.

\title{
How to reference
}

In order to correctly reference this scholarly work, feel free to copy and paste the following:

Vesa Hasu and Heikki Koivo (2012). Automatic Maintenance Routes Based on the Quality Assurance Information, Quality Assurance and Management, Prof. Mehmet Savsar (Ed.), ISBN: 978-953-51-0378-3, InTech, Available from: http://www.intechopen.com/books/quality-assurance-and-management/automaticmaintenance-routes-based-on-quality-assurance-information

\section{INTECH}

open science | open minds

\author{
InTech Europe \\ University Campus STeP Ri \\ Slavka Krautzeka 83/A \\ 51000 Rijeka, Croatia \\ Phone: +385 (51) 770447 \\ Fax: +385 (51) 686166 \\ www.intechopen.com
}

\author{
InTech China \\ Unit 405, Office Block, Hotel Equatorial Shanghai \\ No.65, Yan An Road (West), Shanghai, 200040, China \\ 中国上海市延安西路65号上海国际贵都大饭店办公楼 405 单元 \\ Phone: +86-21-62489820 \\ Fax: +86-21-62489821
}


(C) 2012 The Author(s). Licensee IntechOpen. This is an open access article distributed under the terms of the Creative Commons Attribution 3.0 License, which permits unrestricted use, distribution, and reproduction in any medium, provided the original work is properly cited. 